\title{
Biomasa de hojas caídas y otros indicadores de sustentabilidad en asociaciones de especies forestales con cacao "CCN51" en la zona Central del Litoral ecuatoriano
}

\section{Fallen leaf biomass and other indicators of sustainability in associations of forest species with cocoa "CCN51" in the central zone of the Ecuadorian Coast}

\author{
${ }^{\bullet}$ Gary Ramírez Huila ${ }^{1}$, Emma Torres Navarrete ${ }^{2}$, Nicolás Cruz Rosero ${ }^{1}$, \\ Alexandra Barrera Álvarez ${ }^{2}$, Shirley Alava Ormaza ${ }^{3}$ Marta Jiménez Águila ${ }^{4}$ \\ ${ }^{1}$ Universidad Técnica Estatal de Quevedo. Facultad de Ciencias Ambientales. Carrera de Ingeniería Forestal. \\ Campus "Ingeniero Manuel Haz Álvarez", Av. Quito, km 1.5 vía a Santo Domingo de los Tsáchilas. EC.120301 \\ Quevedo,Ecuador: ^gramirez@uteq.edu.ec; jcruz@uteq.edu.ec \\ ${ }^{2}$ Universidad Técnica Estatal de Quevedo. Facultad de Ciencias Pecuarias. Carrera de Ingeniería Zootecnia. \\ Campus Experimental "La María”. km 7.5 via El Empalme. EC.120301. Quevedo, Ecuador. \\ etorres@uteq.edu.ec; barreraalvarez@yahoo.com \\ ${ }^{3}$ Universidad Técnica Estatal de Quevedo. Unidad de Estudios a Distancia. Escuela de Contador Público Autorizado. Campus \\ “Ingeniero Manuel Haz Álvarez”, Av. Quito, km 1.5 vía a Santo Domingo de los Tsáchilas. EC.120301. \\ Quevedo,Ecuador: alava@uteq.edu.ec \\ ${ }^{4}$ Instituto de Investigaciones Forestales. Calle 174 no. 1723 E/ 17B y 17C Rpto. Siboney, Municipio Playa, \\ La Habana.Cuba.mjimenez@forestales.co.cu
}

Rec.: 9.03.2016. Acept.: 1.07.2016.

Publicado el 30 de diciembre de 2016

\section{Resumen}

Qe realizó el estudio de cuatro especies forestales Cybistax Sdonnell-smithii Rose, Cordia macrantha Chadat, Colubrina arborescens (Mill.) Sarg y Triplaris guayaquilensis Weed, en asociación con Theobroma cacao L. Var. CCN51. El sistema agroforestal estuvo ubicado en Quevedo, provincia de Los Ríos, Ecuador. Los árboles tuvieron seis años y el cacao de cinco años de edad, establecidos en un marco de plantación de 9 × 9 m y 3 × 3 m, respectivamente. Se aplicó un diseño de bloques al azar, con cuatro repeticiones. Las variables evaluadas fueron producción de madera y cacao, sombra, producción de hojas caídas, transferencia de N, P, K, Ca y Mg, nutrientes y contenido de humedad en el suelo. El volumen total de madera para C. donnell-smithii, C. macrantha, $C$. arborescens y T. guayaquilensis fue 46.49; 27.06; 21.03 y $61.37 \mathrm{~m}^{3} \mathrm{ha}^{-1}$, respectivamente. La producción de cacao no presentó diferencias significativas entre asociaciones. Los mayores aportes anuales de biomasa de hojas caídas se produjeron en la asociación $C$. arborescens + cacao con $4079 \mathrm{~kg} \mathrm{ha}^{-1}$, esta asociación realizó la mayor transferencia de N, K, Ca y Mg con 56.45; 29.54; 73.96 y $16.38 \mathrm{~kg} \mathrm{ha}^{-1} \mathrm{a}^{-1}$, respectivamente.

Palabras clave: sistema agroforestal, agroecología, biomasa, cacao var. CCN51.
Gour forest species Cybistax donnell-smithii Rose, Cordia - macrantha Chadat, Colubrina arborescens (Mill.) Sarg and Triplaris guayaquilensis Wedd were studied in association with Theobroma cacao L. Var. CCN51. The agroforestry system was located in Quevedo, province of Los Ríos, Ecuador. The age of the trees was six years, and of cocoa five years, established in a plantation frame of $9 \times 9 \mathrm{~m}$ and $3 \times 3 \mathrm{~m}$, respectively. A random block design with four replicates was applied. The evaluated aspects were: production of wood and cocoa, shade, production of fallen leaves, transfer of N, P, K, $\mathrm{Ca}$ and $\mathrm{Mg}$, nutrients and soil moisture content. The total wood volume for $C$. donnellsmithii, $C$. macrantha, $C$. arborescens and T. guayaquilensis was 46.49; 27.06; 21.03 and $61.37 \mathrm{~m}^{3}$ $\mathrm{ha}^{-1}$, respectively. Cocoa production did not show significant differences between associations. The highest annual biomass contributions of the fallen leaves were produced in the association C. arborescens + cacao with $4079 \mathrm{~kg} \mathrm{ha}^{-1}$, so that it carried out the greatest transfer of $\mathrm{N}, \mathrm{K}, \mathrm{Ca}$ and $\mathrm{Mg}$ with $56.45 ; 29.54 ; 73.96$ and $16.38 \mathrm{~kg} \mathrm{ha}^{-1} \mathrm{a}^{-1}$, respectively.

Key words: Indicators of sustainability, biomass, agroforestry association, cocoa var. CCN51. 


\section{Introducción}

$\mathrm{E}^{\mathrm{s}}$ n sistemas agroforestales el árbol, además de la sombra que puede proporcionar a los cultivos agrícolas, es más eficiente que las plantas herbáceas en la absorción de nutrientes liberados por los horizontes más profundos del suelo (Altieri, 1997; Krishnamurthy y Ávila, 1999).

En el Litoral ecuatoriano el campesino ha desarrollado y practicado varias formas de sistemas agroforestales, entre ellas la asociación de los cultivos tradicionales de exportación como Theobroma cacao L (cacao) con árboles maderables (Torres, 1995; Jaimez, 1997; FAO, 2005). El cacao también se cultiva en asociación con Inga sp (guaba) ya que esta última es una leguminosa mejoradora de los suelos y su sombra beneficia a este cultivo.

Las especies maderables son utilizadas por el valor de su madera y los beneficios adicionales que pueden proporcionar al formar parte de estas asociaciones (Acosta, 1960; Forhier, 1982; Sablón, 1984; Jiménez et al., 2013).

Para complementar el conocimiento tradicional del campesino es necesaria la caracterización de los sistemas agroforestales en el Litoral ecuatoriano. Existe poca información básica sobre indicadores de sustentabilidad en estos sistemas, tales como la producción de biomasa de hojarasca, transferencia de nutrientes, sombra, contenido de humedad en el suelo; además, que en ciertas zonas la hojarasca es la principal vía de ingreso de nutrientes al suelo. Sobre esta base se planteó evaluar los sistemas agroforestales establecidos en la zona Central del Litoral ecuatoriano a partir del estudio de diferentes indicadores cuantitativos tales como producción de la asociación agroforestal, sombra de las especies maderables (\%), hojas caidas de las especies asociadas ( $\mathrm{kg}$ ha año), concentración de nutrientes en hojas caídas de las especies asociadas y su transferencia, contenido de nutrientes y humedad del suelo $(\%)$.

\section{Materiales y métodos}

$\mathrm{E}$ área de investigación está ubicada en la Finca Experimental "La Represa", localizada en el recinto Faita, kilómetro 7,5 de la vía San Carlos, en Quevedo, provincia de Los Ríos, República del Ecuador. Su ubicación geográfica corresponde a 010 03' 18" de latitud sur y 790 25 '24" de longitud oeste. A una altitud de $73 \mathrm{msnm}$, con una temperatura promedio de $24,2^{\circ} \mathrm{C}$, humedad relativa de $77.40 \%$, heliofanía de 823 horas/luz/año, y precipitación media anual de $1537 \mathrm{~mm}$. Zona clasificada como bosque húmedo-Tropical. Los suelos son de textura franco-arcillosa con un $\mathrm{pH}$ de 5.7. Esta investigación se llevó a cabo en un estudio sobre sistemas agroforestales de cuatro especies maderables con cacao de la Unidad de Investigación de la Universidad Técnica Estatal de Quevedo. Las asociaciones agroforestales estudiadas fueron: $C$. donnell smithii Rose + cacao CCN51 C. macrantha Chadat + cacao CCN51; $C$. arborescens (Mill.) Sarg + cacao CCN51; T. guayaquilensis Weed + cacao CCN51.

Para el análisis estadístico de las asociaciones agroforestales se utilizó un diseño de bloques al azar con cuatro tratamientos y cuatro repeticiones. Para la determinación de las diferencias estadísticas entre medias, se aplicó la prueba de Tukey $(\mathrm{p}<0.05)$. Cada especie forestal estuvo dispuesta en un marco de plantación de 9 × $9 \mathrm{~m}$ (123 árboles ha $^{-1}$ ). La unidad experimental constó de nueve árboles maderables y 40 plantas de cacao, var. CCN51. Este último plantado a 3 x $3 \mathrm{~m}$ en y entre las hileras de los árboles maderables (988 plantas ha-1).

En los nueve árboles maderables de cada parcela experimental se midió: diámetro a la altura del pecho (DAP), altura total y comercial, con lo que se calculó el volumen. También se determinó el Incremento Medio Anual, porcentaje de sombra mediante la metodología de Haggar et al. (2001) y Jiménez et al. (2002). La producción de grano seco del cacao CCN51 se registró mensualmente. Además se evaluó la incidencia de Crinipellis perniciosa (Stahel) Singer (escoba de bruja) y Monilla roreri Cif. \& Par. (monilla).

Para evaluar la producción de hojas caídas, en cada parcela se instalaron tres cajas recolectoras de $1 \mathrm{~m}^{2}$ (una bajo la especie maderable y dos bajo el cacao). Mensualmente se determinó el peso seco de las hojas caídas, previo a la eliminación de ramas, cortezas y frutos; el total o una muestra de las mismas fueron colocados en estufa a $70^{\circ} \mathrm{C}$, hasta peso constante (Geigel, 1977; Fassbender, 1987; Quintero y Ataroff, 1998). La estimación del porcentaje de $\mathrm{N}, \mathrm{P}, \mathrm{K}, \mathrm{Ca}$ y $\mathrm{Mg}$ en las hojas caídas y su transferencia se efectuó mediante análisis químico por tratamiento, durante el período lluvioso y seco. En ambos periodos se determinó el contenido de N, P, K, Ca, Mg, M.O y contenido de humedad en el suelo.

\section{Resultados y discusión}

\section{Efecto en las especies maderables}

Diámetro. En las especies maderables, el diámetro presentó diferencias altamente significativas según el test de Tukey $(\mathrm{p}<0.05)$. El mayor diámetro correspondió a T. guayaquilensis con $24.22 \mathrm{~cm}$, seguido de $C$. donnell-smithii con $21.25 \mathrm{~cm}$. Los valores más bajos correspondieron a $C$. macrantha y $C$. arborescens con 18.02 y $17.15 \mathrm{~cm}$, respectivamente.

Altura. En la altura de los árboles también se produjeron diferencias altamente significativas. El mayor valor 
correspondió a T. guayaquilensis con $16.36 \mathrm{~m}$, seguido por C. donnell-smithii con $13.48 \mathrm{~m}$, C. macrantha con $11.64 \mathrm{~m}$ y en el último lugar $C$. arborescens con $10.64 \mathrm{~m}$; equivalente al Incremento medio anual (IMA) de 2.34; 1.92; 1.66 y 1.52 $\mathrm{m} \mathrm{a}^{-1}$, respectivamente.

Volumen. T. guayaquilensis Weed tuvo el mayor volumen por árbol con $0.499 \mathrm{~m}^{3}$, seguido por $C$. donnell-smithii con $0.378 \mathrm{~m}^{3}$, C. macrantha con $0.22 \mathrm{~m}^{3}$ y C. arborescens con $0.171 \mathrm{~m}^{3}$. El volumen por hectárea fue: $T$. guayaquilensis con 61.37; C. donnell-smithii con 46.49; C. macrantha con 27.06 y $C$. arborescens con $21.03 \mathrm{~m}^{3}$ respectivamente; lo que corresponde a un IMA de $12.27 ; 9.29 ; 5.41$ y $4.2 \mathrm{~m}^{3} \mathrm{ha}^{-1}$ $\mathrm{a}^{-1}$, respectivamente. Así mismo en el volumen comercial T. guayaquilensis produjo el mayor volumen con 26.07 $\mathrm{m}^{3} \mathrm{ha}^{-1}$, seguido por $C$. donnell-smithii con $20.78 \mathrm{~m}^{3}$ ha${ }^{1}$, C. macrantha con $13.65 \mathrm{~m}^{3} \mathrm{ha}^{-1}$ y en último lugar $C$. arborescens con $10.45 \mathrm{~m}^{3} \mathrm{ha}^{-1}$, correspondiendo a un IMA de $5.21 ; 4.15 ; 2.73$ y $2.09 \mathrm{~m}^{3} \mathrm{ha}^{-1} \mathrm{a}^{-1}$, respectivamente.

Porcentaje de sombra. El porcentaje de sombra de las especies maderables fue como sigue: $C$. arborescens $60 \%$, C. macrantha $54.62 \%$, C. donnell-smithii $44.38 \%$ y $T$. guayaquilensis $29.11 \%$. El porcentaje de sombra proyectado por T. guayaquilensis se ubicó en el rango de 25 a $30 \%$ propuesto para el cacao (Somarriba, 1994; Somarriba y Beer, 1999 y Álvarez-Carrillo et al., 2012), mientras que $C$. macrantha, $C$. arborescens y $C$. donnell-smithii superaron este rango.

Los árboles de $C$. arborescens, tuvieron un follaje muy denso y la menor altura, lo que disminuyó el ingreso de radiación solar a las plantas de cacao, siendo necesario realizar podas adicionales de acuerdo a su crecimiento. $C$. macrantha y C. donnell-smithii perdieron su follaje durante el período seco, cuando el cacao requirió de mayor protección. Hasta cierto punto esto es una desventaja como especies para sombra frente a $C$. arborescens y $T$. guayaquilensis que mantuvieron el follaje de su copa durante todo el año.
Estas diferencias fenológicas entre las especies maderables estudiadas afectan la posibilidad de mantener la sombra requerida según las necesidades del cacao en cada época.

Producción de hojas caídas en las especies maderables. Se presentaron diferencias altamente significativas en la producción de hojas caídas durante el período lluvioso, seco y total (Cuadro 1). Las especies de mayor producción durante el año fueron $C$. arborescens con $516.00 \mathrm{~g} \mathrm{~m}^{-2}$ y $T$. guayaquilensis con $384.60 \mathrm{~g} \mathrm{~m}^{-2}$ las de menor producción fueron C. macrantha y C. donnell-smithii con 195.50 y $185.20 \mathrm{~g} \mathrm{~m}^{-2}$, respectivamente (Cuadro 1).

La caída de hojas en las especies maderables relacionada con las estaciones climáticas fue variable. La producción fue mayor durante el período seco (Figura 1), concordando con lo planteado por Devineau (1976) y Lastres (1990), quienes manifiestan que la caída de hojarasca tiene un ritmo anual con máximos en la estación seca y mínimos en la lluviosa.

Los arboles de $T$. guayaquilensis produjeron mayor cantidad de hojas durante el período seco, confirmando lo expresado por Suárez et al. (1993), quien señala que esta especie tiene la mayor deposición de sus hojas durante los meses de agosto a septiembre; además manifestó mayor homogeneidad en la producción de hojas durante el año de investigación (Figura 1).

La producción de hojas en kilogramos por hectárea por año de las especies forestales también presentó diferencias altamente significativas. El mayor valor correspondió a C. arborescens con $1997.70 \mathrm{~kg} \mathrm{ha}^{-1} \mathrm{a}^{-1}$, seguido por $T$. guayaquilensis con $1109.88 \mathrm{~kg} \mathrm{ha}^{-1} \mathrm{a}^{-1} \mathrm{y}$ por último $C$. macrantha y C. donnell-smithii con 833.54 y $606.39 \mathrm{~kg} \mathrm{ha}^{-1}$ $\mathrm{a}^{-1}$, respectivamente (Cuadro 1). Los valores obtenidos en las cuatro especies maderables están en el rango determinado por Menéndez y Vilamajó (1988) para nueve especies del bosque tropical siempre verde de Cuba $(70.40-2665.40 \mathrm{~kg}$ $\left.\mathrm{ha}^{-1} \mathrm{a}^{-1}\right)$; a la vez fueron inferiores al reportado por Suárez et al. (1993) para árboles de sombra que produjeron $2000 \mathrm{~kg}$ $h a^{-1} a^{-1}$.

Cuadro 1. Producción de hojas de cuatro especies maderables asociadas con cacao "CCN51"

\begin{tabular}{lcccc}
\hline Especie forestal & $\begin{array}{c}\text { Período lluvioso } \\
\left(\mathbf{g ~ m}^{-2}\right)\end{array}$ & $\begin{array}{c}\text { Período seco } \\
\left(\mathbf{g ~ m}^{-2}\right)\end{array}$ & $\begin{array}{c}\text { Total } \\
\left(\mathbf{g ~ m}^{-2}\right)\end{array}$ & $\left.\mathbf{( K g ~ h a ~}^{-1} \mathbf{~ a}^{-1}\right)$ \\
\hline C. donnell-smithii & $11.00 \mathrm{~b}$ & $174.20 \mathrm{~b}$ & $185.20 \mathrm{c}$ & $606.39 \mathrm{~b}$ \\
C. macrantha & $24.10 \mathrm{~b}$ & $171.40 \mathrm{~b}$ & $195.50 \mathrm{bc}$ & $833.54 \mathrm{~b}$ \\
C. arborescens & $111.70 \mathrm{a}$ & $404.30 \mathrm{a}$ & $516.00 \mathrm{a}$ & $1997.70 \mathrm{a}$ \\
T. guayaquilensis & $164.80 \mathrm{a}$ & $219.70 \mathrm{ab}$ & $384.60 \mathrm{ab}$ & $1109.88 \mathrm{ab}$ \\
\hline E.S. & $\mathbf{1 2 . 7 3}$ & $\mathbf{2 4 . 8 3}$ & $\mathbf{4 7 . 9 2}$ & $\mathbf{1 5 1 . 8 2}$ \\
\hline CV (\%) & $\mathbf{2 8 . 6 7}$ & $\mathbf{3 0 . 9 9}$ & $\mathbf{2 9 . 6 1}$ & $\mathbf{2 6 . 7 1}$ \\
\hline
\end{tabular}

Medias seguidas por la misma letra no presentan diferencias estadísticas (Tukey, p>0.05). 


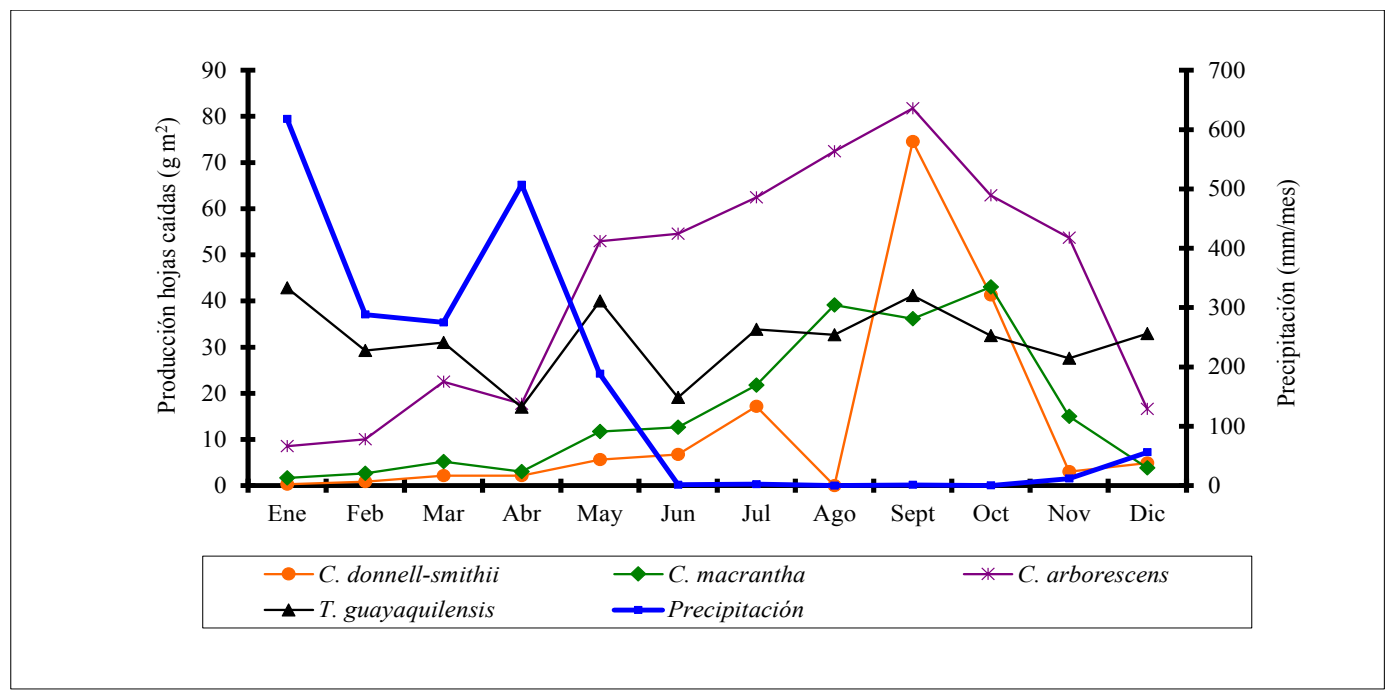

Figura 1. Producción de hojas caídas en $\mathrm{g} / \mathrm{m}^{2} / \mathrm{mes}$ de cuatro especies maderables asociadas con cacao y su relación con las precipitaciones

\section{Producción de semillas e incidencia de enfermedades en el cacao CCN51.}

No se produjeron diferencias estadísticas significativas en la producción de semillas de cacao. La producción estuvo entre $183.30 \mathrm{~kg} \mathrm{ha}^{-1} \mathrm{a}^{-1}$ con C. macrantha y $363.10 \mathrm{~kg} \mathrm{ha}^{-1} \mathrm{a}^{-1}$ con $C$. arborescens.

Según Somarriba y Beer (1999), en sistemas agroforestales los rendimientos moderados de cacao deben ser la meta; en este caso, la producción promedio de semillas de cacao CCN51 obtenida en los tratamientos estudiados no respondió a estas expectativas, más aún si se compara con la producción potencial en plantaciones puras reportada por Crespo y Crespo (1997) quienes aseguran que con una fertilización anual de $600 \mathrm{~g}$ árbol ${ }^{-1}$, riego cada 20 días, poda cada cuatro años, controles fitosanitarios y de malezas se alcanzan rendimientos de $2272 \mathrm{~kg} \mathrm{ha}^{-1} \mathrm{a}^{-1}$ con 1500 plantas por hectárea. Esto sugiere que los sistemas agroforestales deben igualmente ser bien manejados en cuanto a fertilización, riegos y podas.

La presencia de C. perniciosa (Stagel) Singer (escoba de bruja) en los períodos lluvioso y seco, fue entre ligera y moderada. Mientras que la afectación por $M$. roreri Cif. \& Par. (monilla) estuvo entre 2.25 y $3.25 \%$, porcentajes bajos si se comparan con el $30 \%$ que puede alcanzar en el cacao CCN51 (Crespo y Crespo, 1997).

\section{Producción de hojas caídas del cacao CCN51}

La producción de hojas caídas del cacao no presentó diferencias estadísticas; estuvo entre $272.40 \mathrm{~g} \mathrm{~m}^{2}$ en el tratamiento C. arborescens + cacao y $309.40 \mathrm{~g} \mathrm{~m}^{-2}$ en $C$. donnell-smithii + cacao; lo que equivale a $1692.96 \mathrm{y}$ $2404.54 \mathrm{~kg} \mathrm{ha}^{-1} \mathrm{a}^{-1}$, respectivamente (Cuadro 2). La caída de hojas en el cacao no fue influenciada por la presencia de las especies forestales o sombreadoras, este fenómeno interactuó directamente con los factores ambientales como la lluvia y la sequía (Figura 2). La alta producción de hojas del cacao, corrobora que éste, al ser un cultivo de plantación permanente constituye un sistema de importancia como reforestador, ya que aporta a los ecosistemas gran cantidad de materia orgánica a través de la hojarasca; esta característica favorece la conservación, fertilidad y el desarrollo de la micro y macrofauna del suelo (Schalatter et al., 2003; Schalatter et al., 2006).

\section{Transferencia de nutrientes por hojas caídas de las especies forestales}

En el Cuadro 3 se observan, los contenidos de N, P, K, $\mathrm{Ca}$ y Mg en las hojas caídas, así como la transferencia de los mismos por las especies maderables. C. arborescens se destaca por la mayor transferencia de nutrientes, menos en $P$ donde sobresale $C$. macrantha.

\section{Transferencia de nutrientes por hojas caídas del cacao CCN51}

En el Cuadro 4 se observan los contenidos de N, P, K, $\mathrm{Ca}$ y $\mathrm{Mg}$ en las hojas caídas, así como la transferencia de los mismos por el cacao en cada sistema. Resaltó el cacao asociado con C. donnell-smithii y T. guayaquilensis por mayor transferencia de nutrientes.

\section{Transferencia de nutrientes por sistema agroforestal}

La transferencia total de nitrógeno $(\mathrm{N})$ de la fracción foliar de las especies forestales y el cacao CCN51 estuvo en un rango de 37.12 a $56.45 \mathrm{~kg} \mathrm{ha}^{-1} \mathrm{a}^{-1}$ en C.macranta + cacao y $C$. arborescens + cacao en su orden; estos aportes fueron menores a los reportados por Jaimez y Franco (1999), para asociaciones agroforestales en Mérida, Venezuela con una transferencia de $\mathrm{N}$ entre $79.70 \mathrm{~kg} \mathrm{ha}^{-1} \mathrm{a}^{-1}$ en el sistema Annona muricata + Persea americana + cacao hasta $129.50 \mathrm{~kg} \mathrm{ha}^{-1} \mathrm{a}^{-1}$ para el sistema Pouteria sapota + cacao. La transferencia total 
Cuadro 2. Producción de hojas del cacao "CCN51" asociado con cuatro especies forestales

\begin{tabular}{|c|c|c|c|c|}
\hline Tratamiento & $\begin{array}{c}\text { Período lluvioso } \\
\left(\mathrm{g} \mathrm{m}^{-2}\right) \\
\end{array}$ & $\begin{array}{c}\text { Período seco } \\
\left(\mathrm{g} \mathrm{m}^{-2}\right) \\
\end{array}$ & $\begin{array}{r}\text { Total } \\
\left(\mathrm{g} \mathrm{m}^{-2}\right) \\
\end{array}$ & $\left(\mathrm{Kg} \mathrm{ha}^{-1} \mathbf{a}^{-1}\right)$ \\
\hline C. donnell-smithii + cacao & $29.30 \mathrm{a}$ & $280.10 \mathrm{a}$ & $309.40 \mathrm{a}$ & $2404.54 \mathrm{a}$ \\
\hline C. macrantha + cacao & $26.10 \mathrm{a}$ & $255.40 \mathrm{a}$ & $281.40 \mathrm{a}$ & $1692.96 \mathrm{a}$ \\
\hline C. arborescens + cacao & $32.50 \mathrm{a}$ & $242.20 \mathrm{a}$ & $272.40 \mathrm{a}$ & $2081.63 \mathrm{a}$ \\
\hline T. guayaquilensis + cacao & $36.10 \mathrm{a}$ & $275.30 \mathrm{a}$ & $286.80 \mathrm{a}$ & $2126.68 \mathrm{a}$ \\
\hline E.S. & 11.65 & 19.62 & 14.33 & 197.90 \\
\hline CV (\%) & 25.18 & 14.91 & 9.97 & 19.06 \\
\hline
\end{tabular}

Medias seguidas por la misma letra no presentan diferencias estadísticas (Tukey, p>0.05).

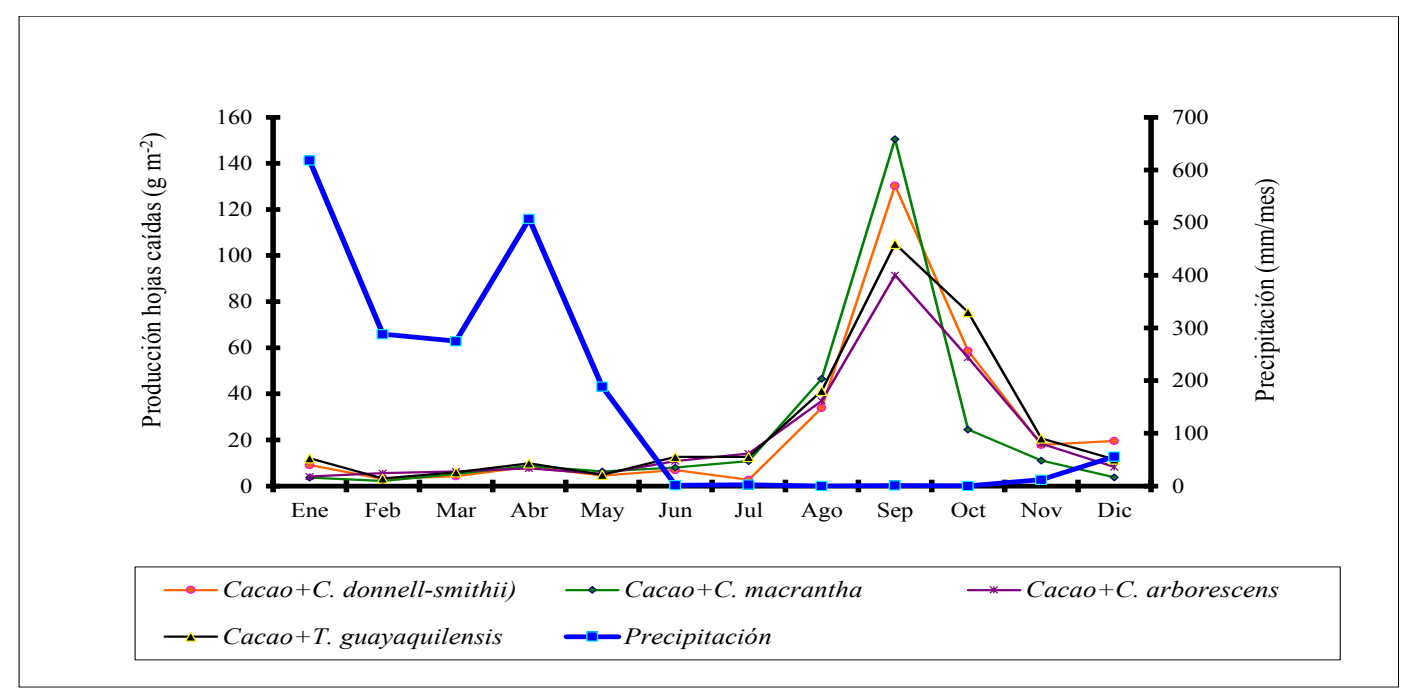

Figura 2. Producción de hojas caídas en $\mathrm{g} / \mathrm{m}^{2} / \mathrm{mes}$ del cacao CCN51 asociado con cuatro especies maderables y su relación con las precipitaciones

de fósforo $(\mathrm{P})$ por la fracción foliar de las especies forestales y el cacao, estuvo entre $3.03 \mathrm{~kg} \mathrm{ha}^{-1} \mathrm{a}^{-1}$ en el sistema C. donnellsmithii + cacao y $5.87 \mathrm{~kg} \mathrm{ha}^{-1} \mathrm{a}^{-1}$ en el sistema C. macrantha + cacao. Jaimez y Franco (1999) determinaron una transferencia de P por la fracción foliar de las asociaciones agroforestales entre 9.90 y $11.20 \mathrm{~kg} \mathrm{ha}^{-1} \mathrm{a}^{-1}$ para sistemas agroforestales con cacao; mientras que Fassbender et al. (1988) reportaron 13.80 y $8.80 \mathrm{~kg} \mathrm{ha}^{-1} \mathrm{a}^{-1}$ para los sistemas Cordia alliodora + cacao y Erythrina poeppigiana + cacao, respectivamente; Aranguren (1979) encontró una alta transferencia con $22.70 \mathrm{~kg} \mathrm{ha}^{-1} \mathrm{a}^{-1}$, mientras que Santana et al. (1990) reportaron en Bahía - Brasil valores que oscilaron entre 5 y $10.70 \mathrm{~kg} \mathrm{ha}^{-1} \mathrm{a}^{-1}$.

El aporte total de potasio $(\mathrm{K})$ por las asociaciones estudiadas, estuvo entre 9.94 y $29.54 \mathrm{~kg} \mathrm{ha}^{-1} \mathrm{a}^{-1}$ para los sistemas $C$. macrantha + cacao y C. arborescens + cacao, respectivamente. En Bahía, Brasil, se reportaron rangos variables de transferencia de $\mathrm{K}$ en sistemas agroforestales, entre 29.90 y $73 \mathrm{~kg} \mathrm{ha}^{-1} \mathrm{a}^{-1}$ (Santana et al., 1990); mientras que Jaimez y Franco (1999) determinaron un alto aporte de este elemento a través de las hojas en el sistema $P$. zapota + cacao con $61.10 \mathrm{~kg} \mathrm{ha}^{-1} \mathrm{a}^{-1}$. La transferencia total de calcio (Ca) por las asociaciones agroforestales estudiadas estuvo entre 34.79 $\mathrm{kg} \mathrm{ha}^{-1} \mathrm{a}^{-1}$ en C. macrantha + cacao y $73.96 \mathrm{~kg} \mathrm{ha}^{-1} \mathrm{a}^{-1}$ en la asociación C. arborescens + cacao. Jaimez y Franco (1988) reportaron transferencias de $\mathrm{Ca}$ en sistemas agroforestales con cacao entre 84 y $162 \mathrm{~kg} \mathrm{ha}^{-1} \mathrm{a}^{-1}$. Para comparación, valores de transferencias de $\mathrm{Ca}$ en sistemas agroforestales con cacao comprendidos entre 81 y $100 \mathrm{~kg} \mathrm{ha}^{-1} \mathrm{a}^{-1}$ fueron determinados en el estado de Bahía - Brasil (Santana et al., 1990).

$\mathrm{La}$ transferencia total de magnesio $(\mathrm{Mg})$ en estas asociaciones agroforestales estuvo entre $13.98 \mathrm{~kg} \mathrm{ha}^{-1} \mathrm{a}^{-1}$ en C. macrantha + cacao y $16.38 \mathrm{~kg} \mathrm{ha}^{-1} \mathrm{a}^{-1}$ en $C$. arborescens + cacao, valores inferiores a los reportados por Jaimez y Franco (1999), quienes obtuvieron aportes de $\mathrm{Mg}$ de $31.70 \mathrm{~kg} \mathrm{ha}^{-1}$ $\mathrm{a}^{-1}$ en el sistema $P$. americana + cacao, $17 \mathrm{~kg} \mathrm{ha}^{-1} \mathrm{a}^{-1}$ para P. sapota + cacao y $23.60 \mathrm{~kg} \mathrm{ha}^{-1} \mathrm{a}^{-1}$ para A. muricata $+P$. americana + cacao; Asimismo, Cardona-Calle y SadeghianKhalajabadi (2005) publicaron retornos de 27.31 y $30.66 \mathrm{~kg}$ 
Cuadro 3. Nutrientes en hojas caídas (\%) y su transferencia $\left(\mathrm{kg} \mathrm{ha} \mathrm{a}^{-1}\right)$ en las cuatro especies forestales asociadas con cacao "CCN51"

\begin{tabular}{|c|c|c|c|c|}
\hline Nutrientes & Especie forestal & $\begin{array}{c}\text { Periodo Lluvioso } \\
\text { (\%) }\end{array}$ & $\begin{array}{c}\text { Periodo Seco } \\
(\%)\end{array}$ & $\begin{array}{c}\text { Transferencia } \\
\left(\mathrm{Kg} \mathrm{ha}^{-1} \mathbf{a}^{-1}\right)\end{array}$ \\
\hline \multirow{6}{*}{$\mathrm{N}$} & C. donnell-smithii & 1.40 & 2.30 & $13.63 \mathrm{ab}$ \\
\hline & C. macrantha & 1.70 & 1.50 & $12.70 \mathrm{ab}$ \\
\hline & C. arborescens & 1.70 & 1.60 & $32.05 \mathrm{a}$ \\
\hline & T. guayaquilensis & 1.20 & 0.70 & $10.10 \mathrm{~b}$ \\
\hline & E.S & - & - & 2.02 \\
\hline & CV (\%) & - & - & 24.44 \\
\hline \multirow{6}{*}{$\mathrm{P}$} & C. donnell-smithii & 0.12 & 0.10 & $0.61 \mathrm{c}$ \\
\hline & C. macrantha & 0.34 & 0.56 & $4.44 \mathrm{a}$ \\
\hline & C. arborescens & 0.23 & 0.11 & $2.68 \mathrm{~b}$ \\
\hline & T. guayaquilensis & 0.11 & 0.07 & $0.96 \mathrm{c}$ \\
\hline & E.S & - & - & 0.25 \\
\hline & CV (\%) & - & - & 23.60 \\
\hline \multirow{6}{*}{$\mathrm{K}$} & C. donnell-smithii & 0.46 & 1.35 & $7.86 \mathrm{~b}$ \\
\hline & C. macrantha & 0.47 & 0.28 & $2.52 \mathrm{c}$ \\
\hline & C. arborescens & 0.74 & 1.13 & $20.66 \mathrm{a}$ \\
\hline & T. guayaquilensis & 1.10 & 1.10 & $12.14 \mathrm{ab}$ \\
\hline & E.S & - & - & 2.23 \\
\hline & CV (\%) & - & - & 28.97 \\
\hline \multirow{6}{*}{$\mathrm{Ca}$} & C. donnell-smithii & 2.31 & 1.61 & $10.02 b$ \\
\hline & C. macrantha & 1.87 & 3.06 & $24.27 \mathrm{ab}$ \\
\hline & C. arborescens & 1.55 & 1.73 & $33.43 \mathrm{a}$ \\
\hline & T. guayaquilensis & 0.80 & 1.29 & $11.92 b$ \\
\hline & E.S & - & - & 3.60 \\
\hline & $\mathrm{CV}(\%)$ & - & - & 24.24 \\
\hline \multirow{6}{*}{$\mathrm{Mg}$} & C. donnell-smithii & 0.61 & 0.21 & $1.42 \mathrm{a}$ \\
\hline & C. macrantha & 0.36 & 0.32 & $2.70 \mathrm{a}$ \\
\hline & C. arborescens & 0.27 & 0.17 & $3.78 \mathrm{a}$ \\
\hline & T. guayaquilensis & 0.17 & 0.19 & $2.00 \mathrm{a}$ \\
\hline & E.S. & - & - & 0.65 \\
\hline & $\mathrm{CV}(\%)$ & - & - & 23.40 \\
\hline
\end{tabular}

Medias seguidas por la misma letra no presentan diferencias estadísticas (Tukey, p>0.05). 
Cuadro 4. Nutrientes en hojas caídas (\%) y su transferencia $\left(\mathrm{kg}\right.$ ha $\left.\mathrm{a}^{-1}\right)$ en el cacao "CCN51" asociado con cuatro especies forestales

\begin{tabular}{|c|c|c|c|c|}
\hline Nutrientes & Especie forestal & $\begin{array}{c}\text { Periodo Lluvioso } \\
(\%)\end{array}$ & $\begin{array}{c}\begin{array}{c}\text { Periodo seco } \\
(\%)\end{array} \\
\end{array}$ & $\begin{array}{c}\text { Transferencia } \\
\left(\mathrm{Kg} \mathrm{ha}^{-1} \mathbf{a}^{-1}\right) \\
\end{array}$ \\
\hline \multirow{6}{*}{$\mathrm{N}$} & C. donnell-smithii + cacao & 1.60 & 1.20 & $29.75 \mathrm{a}$ \\
\hline & C. macrantha + cacao & 1.20 & 1.50 & $24.92 \mathrm{a}$ \\
\hline & C. arborescens + cacao & 1.40 & 0.70 & $24.40 \mathrm{a}$ \\
\hline & T. guayaquilensis + cacao & 1.70 & 1.30 & $31.09 \mathrm{a}$ \\
\hline & E.S. & & & 2.86 \\
\hline & CV (\%) & & & 20.80 \\
\hline \multirow{6}{*}{$\mathrm{P}$} & C. donnell-smithii + cacao & 0.11 & 0.10 & $2.42 \mathrm{ab}$ \\
\hline & C. macrantha + cacao & 0.09 & 0.08 & $1.36 \mathrm{~b}$ \\
\hline & C. arborescens + cacao & 0.10 & 0.09 & $1.58 \mathrm{ab}$ \\
\hline & T. guayaquilensis + cacao & 0.10 & 0.11 & $2.50 \mathrm{a}$ \\
\hline & E.S. & & & 0.18 \\
\hline & CV (\%) & & & 18.88 \\
\hline \multirow{6}{*}{ K } & C. donnell-smithii + cacao & 0.36 & 0.43 & $10.18 \mathrm{a}$ \\
\hline & C. macrantha + cacao & 0.37 & 0.25 & $4.42 \mathrm{~b}$ \\
\hline & C. arborescens + cacao & 0.21 & 0.55 & $8.88 \mathrm{ab}$ \\
\hline & T. guayaquilensis + cacao & 0.41 & 0.45 & $10.27 \mathrm{a}$ \\
\hline & E.S. & & & 0.80 \\
\hline & CV (\%) & & & 19.00 \\
\hline \multirow{6}{*}{$\mathrm{Ca}$} & C. donnell-smithii + cacao & 1.99 & 2.03 & $48.70 \mathrm{a}$ \\
\hline & C. macrantha + cacao & 2.33 & 2.40 & $40.52 \mathrm{a}$ \\
\hline & C. arborescens + cacao & 1.99 & 2.37 & $40.53 \mathrm{a}$ \\
\hline & T. guayaquilensis + cacao & 1.74 & 2.05 & $46.50 \mathrm{a}$ \\
\hline & E.S. & & & 2.23 \\
\hline & CV (\%) & & & 19.25 \\
\hline \multirow{6}{*}{$\mathrm{Mg}$} & C. donnell-smithii + cacao & 0.47 & 0.66 & $14.42 \mathrm{a}$ \\
\hline & C. macrantha + cacao & 0.54 & 0.68 & $11.28 \mathrm{a}$ \\
\hline & C. arborescens + cacao & 0.60 & 0.74 & $12.60 \mathrm{a}$ \\
\hline & T. guayaquilensis + cacao & 0.42 & 0.71 & $13.61 \mathrm{a}$ \\
\hline & E.S. & & & 1.31 \\
\hline & CV $(\%)$ & & & 19.12 \\
\hline
\end{tabular}

Medias seguidas por la misma letra no presentan diferencias estadísticas (Tukey, p>0.05). 
Cuadro 5. Caracterización química de los suelos y su interpretación correspondiente a las cuatro asociaciones agroforestales

\begin{tabular}{|c|c|c|c|c|c|}
\hline \multirow[b]{2}{*}{ Nutriente } & \multirow[b]{2}{*}{ Tratamiento } & \multicolumn{2}{|c|}{ Período Lluvioso } & \multicolumn{2}{|c|}{ Período seco } \\
\hline & & $\begin{array}{c}\text { Contenido } \\
(\%) \\
\end{array}$ & $\begin{array}{c}\text { Interpretación } \\
* \\
\end{array}$ & $\begin{array}{c}\text { Contenido } \\
(\%) \\
\end{array}$ & $\begin{array}{c}\text { Interpretación } \\
* \\
\end{array}$ \\
\hline \multirow{6}{*}{$\begin{array}{c}\mathrm{N} \\
\mathrm{ppm}\end{array}$} & C. donnell-smithii + cacao & $25.25 \mathrm{a}$ & B & $12.00 \mathrm{a}$ & B \\
\hline & C. macrantha + cacao & $22.75 \mathrm{a}$ & B & $9.75 \mathrm{a}$ & B \\
\hline & C. arborescens + cacao & $26.00 \mathrm{a}$ & B & $18.00 \mathrm{a}$ & B \\
\hline & T. guayaquilensis + cacao & $10.50 \mathrm{a}$ & B & $16.75 \mathrm{a}$ & B \\
\hline & E.S & 2.46 & - & 3.69 & - \\
\hline & $\mathrm{CV} \%$ & 23.36 & - & 22.38 & - \\
\hline \multirow{6}{*}{$\begin{array}{c}\mathrm{P} \\
\mathrm{ppm}\end{array}$} & C. donnell-smithii + cacao & $68.00 \mathrm{~b}$ & A & $81.75 \mathrm{a}$ & A \\
\hline & C. macrantha + cacao & $93.50 \mathrm{a}$ & A & $68.50 \mathrm{a}$ & A \\
\hline & C. arborescens + cacao & $80.50 \mathrm{ab}$ & A & $73.00 \mathrm{a}$ & A \\
\hline & T. guayaquilensis + cacao & $86.00 \mathrm{a}$ & A & $72.75 \mathrm{a}$ & A \\
\hline & E.S & 4.30 & - & 18.37 & - \\
\hline & $\mathrm{CV} \%$ & 10.49 & - & 29.67 & - \\
\hline \multirow{6}{*}{$\begin{array}{c}\mathrm{K} \\
\mathrm{meq} / 100 \mathrm{~mL}\end{array}$} & C. donnell-smithii + cacao & $0.35 \mathrm{c}$ & M & $0.47 \mathrm{a}$ & A \\
\hline & C. macrantha + cacao & $0.44 b$ & A & $0.57 \mathrm{a}$ & $\mathrm{A}$ \\
\hline & C. arborescens + cacao & $0.61 \mathrm{a}$ & $\mathrm{A}$ & $0.62 \mathrm{a}$ & A \\
\hline & T. guayaquilensis + cacao & $0.58 \mathrm{a}$ & $\mathrm{A}$ & $0.59 \mathrm{a}$ & $\mathrm{A}$ \\
\hline & E.S & 0.02 & - & 0.10 & - \\
\hline & $\mathrm{CV} \%$ & 5.11 & - & 27.67 & - \\
\hline \multirow{6}{*}{$\begin{array}{c}\mathrm{Ca} \\
\mathrm{meq} / 100 \mathrm{~mL}\end{array}$} & C. donnell-smithii + cacao & $11.00 \mathrm{a}$ & A & $11.25 \mathrm{a}$ & A \\
\hline & C. macrantha + cacao & $11.00 \mathrm{a}$ & A & $10.00 \mathrm{a}$ & A \\
\hline & C. arborescens + cacao & $10.25 \mathrm{a}$ & A & $10.50 \mathrm{a}$ & A \\
\hline & T. guayaquilensis + cacao & $10.50 \mathrm{a}$ & A & $9.00 \mathrm{a}$ & A \\
\hline & E.S & 0.31 & - & 0.97 & - \\
\hline & $\mathrm{CV} \%$ & 5.89 & - & 19.24 & - \\
\hline \multirow{6}{*}{$\begin{array}{c}\mathrm{Mg} \\
\mathrm{meq} / 100 \mathrm{~mL}\end{array}$} & C. donnell-smithii + cacao & $1.65 \mathrm{a}$ & B & $1.52 \mathrm{a}$ & B \\
\hline & C. macrantha + cacao & $1.52 \mathrm{ab}$ & B & $1.42 \mathrm{a}$ & B \\
\hline & C. arborescens + cacao & $1.52 \mathrm{ab}$ & B & $1.42 \mathrm{a}$ & B \\
\hline & T. guayaquilensis + cacao & $1.45 \mathrm{~b}$ & B & $1.35 \mathrm{a}$ & B \\
\hline & E.S & 0.03 & - & 0.08 & - \\
\hline & $\mathrm{CV} \%$ & 3.91 & - & 12.01 & - \\
\hline \multirow{6}{*}{$\begin{array}{l}\text { M.O } \\
(\%)\end{array}$} & C. donnell-smithii + cacao & $2.82 \mathrm{a}$ & B & $3.12 \mathrm{a}$ & M \\
\hline & C. macrantha + cacao & $2.92 \mathrm{a}$ & B & $2.85 \mathrm{a}$ & B \\
\hline & C. arborescens + cacao & $3.00 \mathrm{a}$ & M & $2.92 \mathrm{a}$ & B \\
\hline & T. guayaquilensis + cacao & $2.70 \mathrm{a}$ & B & $2.70 \mathrm{a}$ & B \\
\hline & E.S. & 0.08 & - & 0.17 & - \\
\hline & CV $(\%)$ & 6.24 & - & 12.27 & - \\
\hline
\end{tabular}

Medias seguidas por la misma letra no presentan diferencias estadísticas (Tukey, $\mathbf{p}>\mathbf{0 . 0 5}$ ). $\quad * \mathrm{~B}=$ bajo, $\mathrm{M}=$ medio, $\mathrm{A}=$ alto 
ha $^{-1} \mathrm{a}^{-1}$ en los agroecosistemas de cafetales bajo sombra con Chinchiná y Albán, respectivamente; Fassbender et al. (1988) por su parte, reportan un promedio de $50.10 \mathrm{~kg} \mathrm{ha}^{-1} \mathrm{a}^{-1}$ para los sistemas E. poeppigiana + cacao y C. alliodora + cacao. El sistema $C$. arborescens + cacao ocupó el primer lugar en transferencia de $\mathrm{N}, \mathrm{K}$, Ca y $\mathrm{Mg}$, debido fundamentalmente a la mayor cantidad de hojas que produjeron los árboles de $C$. arborescens y al importante aporte del cacao. Los elementos que más necesita la planta de cacao para su desarrollo son $\mathrm{N}$, K y Ca, de estos el $\mathrm{K}$ siempre es requerido en mayores cantidades (Mite y Motato, 1993; Jaimez y Franco, 1999); en este estudio el sistema $C$. arborescens + cacao transfirió las mayores cantidades de N, K y Ca, con 56.45; 29.54 y 73.96 $\mathrm{kg} \mathrm{ha}^{-1} \mathrm{a}^{-1}$, respectivamente.

\section{Nutrientes en el suelo}

La caracterización química de los suelos, correspondiente a las cuatro asociaciones agroforestales fue diferente $(\mathrm{p}<0.05)$ en los contenidos, sin embargo, es similar para los dos períodos (Cuadro 5). El potencial Hidrógeno $(\mathrm{pH})$ en el suelo de los tratamientos estudiados y fuera del área de investigación estuvo entre 5.60-5.90; valores interpretados como medianamente Ácidos (MeAc).

\section{Contenido de humedad en el suelo}

El contenido de humedad en el suelo, evaluado durante los meses secos manifestó diferencias $(p<0.05)$. En los tratamientos $C$. arborescens + cacao y $T$. guayaquilensis + cacao se produjeron los mayores valores. Los menores porcentajes se obtuvieron en $C$. macrantha + cacao y $C$. donnell-smithii + cacao (Cuadro 6).

Los contenidos de humedad determinados para los cuatro tratamientos están por debajo del nivel adecuado que debe tener el suelo, según Suárez et al. (1993), la cantidad de agua en el suelo es adecuada en un 30 a $40 \%$ de humedad, con un nivel alto constante, pues, los árboles de sombra transpiran mayor cantidad de agua que los de cacao y cuando alcanzan un desarrollo completo dejan caer ramas o se defolian totalmente durante los períodos secos, a consecuencia de esto, los árboles de cacao sufren una excesiva transpiración, a menos que el suelo tenga una capacidad realmente alta y constante, para proporcionar humedad. En este sentido, los valores más altos (17.29 y 17.34\%) son de las especies C. arborescens y T. guayaquilensis; que presentaron una mayor caída de hojas, produciendo mayor cobertura del suelo, evitando mayor evaporación de agua, reteniendo agua y nutrientes para las plantas; de acuerdo a Branson et al. (1981); Waring y Running (1998); Nebel y Wrigth (1999); SEMARNAT (2003) y Martínez-González et al. (2010), la hojarasca que se desprende de los árboles, forman materia orgánica, por lo que los suelos con mayor vegetación que tienen una mayor cobertura del suelo y mayor materia orgánica podrían tener menor erosión y mejor conservación de su humedad.
Cuadro 6. Promedio mensual del contenido de humedad del suelo de las asociaciones agroforestales durante el período seco

\begin{tabular}{lc} 
Tratamiento & Contenido de humedad \\
\hline C. donnell-smithii + cacao & $13.5 \mathrm{~b}$ \\
C. macrantha + cacao & $14.28 \mathrm{~b}$ \\
C. arborescens + cacao & $17.29 \mathrm{a}$ \\
T. guayaquilensis + cacao & $17.34 \mathrm{a}$ \\
\hline E.S. & $\mathbf{0 . 6 7}$ \\
\hline CV $(\%)$ & $\mathbf{8 . 6 3}$ \\
\hline
\end{tabular}

Medias seguidas por la misma letra no presentan diferencias estadísticas (Tukey, $\mathrm{p}>\mathbf{0 . 0 5}$ ).

\section{Conclusiones}

T os maderables T. guayaquilensis y C. donnel-smithii, tuvieron la mayor producción de madera, siendo similares en la producción de hojas caídas, de semillas e incidencia de $C$. perniciosa y $M$. roreri en el cacao.

El mayor porcentaje de sombra lo produjo $C$. arborescens. Los mayores aportes anuales de biomasa de hojas caídas se dió en la asociación C. arborescens + cacao, transfiriendo mayor cantidad de $\mathrm{N}, \mathrm{K}$ y Ca, mientras que el contenido de materia orgánica fue igual en todas las asociaciones.

Los árboles de T. guayaquilensis y C. arborescens por su alta producción de hojarasca y follaje permanente durante todo el año realizaron una mayor cobertura, acumulando mayor contenido de humedad en el suelo.

\section{Bibliografía}

Acosta, M. (1960). Maderas económicas del Ecuador y sus usos. Casa de la Cultura Ecuatoriana. Quito, EC. 69 p.

Altieri, M. (1997). Agroecología. Bases para una agricultura sustentable. CLADES. CIED. Secretariado Rural Perú, Bolivia. Lima, PE. 511p

Álvarez-Carrillo, F., Rojas-Molina, J., Suarez-Salazar, J. (2012). Simulación de arreglos agroforestales de cacao como una estrategia de diagnóstico y planificación para productores. Corpoica Cienc. Tecnol. Agropecu. 13(2): 145-150

Aranguren, J. (1979). Contribución de la caída de hojarasca al ciclo de nutrientes en cultivos bajo árboles de sombra. Tesis Mag. Sc. Instituto Venezolano de Investigaciones Científicas. Caracas, VE. 285 p

Beer, J.(1999). Theobroma cacao: un cultivo "agroforestal". Revista Agroforestería en las Américas 6(22). 
Branson, FA., Gifford, GF., Renard, KG., and Hadley, RF. (1981). Rangeland Hydrology. Second Edition. Dubuque, USA: Kendall/Hunt Publishing Company, pp. 37-41, 47-92.

Cardona-Calle, DA., Sadeghian-Khalajabadi, S. (2005). Ciclo de nutrimentos y actividad microbiana en cafetales a libre exposición solar y con sombrío de Inga spp. Cenicafé 56(2): 127-141.

Crespo, E., y Crespo, F. (1997). Cultivo y beneficio del cacao CCN51. El Conejo. Quito, EC. 133p

Devineau, J. (1976). Dones preliminaires sur la litiére et la chute des feuilles dans quelques formations forestieres semi-desidues de moyenne cote-d'Ivoire. Oecol. Plant. 11(4): 375-395.

FAO. (2005). Estudio de tendencias y perspectivas del Sector Forestal en América Latina. Documento de Trabajo. Informe nacional Ecuador. Dirección Nacional Forestal. Roma. 67 p.

Fassbender, H. (1987). Modelos edafológicos de sistemas agroforestales. CATIE. Turrialba, CR. 184 p

Fassbender, H., Alpizar; L., Heuveldop, J., Folster, H., and Enriquez, G. (1988). Modeling agroforestry systems of cacao (T. cacao) with laurel (C. Alliodora) and poro (E. Poeppigiana) in Costa Rica. III Cycles of matter and nutrients. Agroforestry Systems 6:49-62

Forhier, L. (1982). Importancia de sistemas agroforestales en Costa Rica. Agronomía Costarricence. CR. 5p.

Geigel, F. (1977). Materia Orgánica y, nutrientes devueltos al suelo mediante la hojarasca de diversas especies forestales. Revista Forestal Baracoa 7(3-4): 15-38

Haggar, J., Schibli, C., Staver, C. (2001). ¿Cómo manejar árboles de sombra en cafetales? Revista Agroforestería en las Américas 8(29): 37-41

Jaimez, R. (1997). Aportes de macronutrientes en agrosistemas de cacao con frutales en la región de Tucaní. Universidad de Los Andes. Facultad de Ciencias Forestales y Ambientales. Centro de Estudios de Forestal. Mérida, Venezuela. 103 p.

Jaimez, R., y Franco, W. (1999). Producción de hojarasca, aporte en nutrientes y descomposición en sistemas agroforestales de cacao y frutales. Universidad de Los Andes. Mérida, Venezuela. 8 p

Jiménez, J., Kramer, H., Aguirre, O. (2002). Bestandesuntersuchungen in einem ungleichaltrigen Tannen-, Douglasien-, KiefernNaturbestand Nordostmexikos. Allgemeine Forst und Jagdzeitung 173: 47-55.

Jiménez, M., Calzadilla, E., Renda, A., Reyes, F., Mosquera, A., Merlán, G., Farril, A., Curbelo, S., Fleitas, Y., Reyes, J., González, M., y Friol, P. (2013). Sistemas agroforestales en Cuba, treinta años de experiencia. Agricultura Orgánica, 19(2): 14-17.

Krishnamurthy, L., y Avila, M. (1999). Agroforestería básica. FAO. Red de Formación Ambiental para
América Latina y el Caribe. México, D.F. 340 p

Lastres, L. (1990). Dinámica de las reservas orgánicas y energéticas de la hojarasca de un bosque tropical semideciduo en Cuba. Tesis Doctor Ciencias Biológicas. Academia de Ciencias de Cuba. Ciudad de la Habana, CU. 32 p

Martínez-González, F., Sosa-Pérez, F., Ortiz-Medel, J. (2010). Comportamiento de la humedad del suelo con diferente cobertura vegetal en la Cuenca La Esperanza. Tecnología y Ciencias del Agua, 1(4): 89103.

Menéndez, L. \& Vilamajó, D. (1988). El banco de semillas. En: Ecología de los bosques siempreverdes de la Sierra del Rosario, Cuba. Proyecto MAB No. 1, 19741987. (Eds. R.A. Herrera, Leda Menéndez, María Rodríguez \& Elisa García). ROSTLAC, Montevideo. p. 261

Mite, F., y Motato, N. (1993). Suelos y fertilizantes. In Manual del cultivo de cacao. Instituto Nacional Autónomo de Investigaciones Agropecuarias. EC. p. 70-74

Nebel, BJ., y Wrigth, RT. (1999). Ciencias ambientales, "Ecología y desarrollo sostenible". Sexta edición. México, D.F.: Editorial Prentice Hall Hispanoamericana, pp. 211-230.

Quintero, JS. y Ataroff, M. (1998). Contenido y flujo de nitrógeno en la biomasa y hojarasca de un cafetal a plena exposición solar en los Andes venezolanos. Rev. Fac. Agron. (LUZ). 15(6): 501-514

Sablón, A. (1984). Dendrología. Ministerio de Educación Superior. Pueblo y Educación. Ciudad de la Habana, CU. p 117-119

Santana, M., Cabala-Rosand, P., y Serodio, E. (1990). Reciclage de nutrientes em agrossistemas de cacau. Agrotrópica (Brasil) 2(2): 68-74

Schalatter, J., Gerding, V., Calderón, S. (2006). Aporte de la hojarasca al ciclo biogeoquímico en plantaciones de Eucalyptus nitens. Chile, Revista Bosque 27(2): 115-125.

Schalatter, J., Grez, R., Gerding, V. (2003). Manual para el reconocimiento de suelos. Universidad Austral de Chile, Valdivia, Chile. 1148 p.

SEMARNAT. (2003). Hombre naturaleza. Cruzada por los bosques y el agua. Saber para proteger. Introducción a los servicios ambientales. México, D.F.: Secretaría de Medio Ambiente y Recursos Naturales, pp. 5-16.

Somarriba, E. (1994). Maderables como alternativa para la sustitución de sombra de cacaotales establecidos. El concepto. CATIE. Turrialba, CR. 30p

Somarriba, E. y Beer, J. (1999). Sistemas agroforestales con cacao en Costa Rica y Panamá. Revista Agroforestería en las Américas 6(22): 7-11

Suárez, C., Mite, F., Páliz, V., Sotomayor, I., Vera, J., Moreira, M. (1993). Manual de cultivo de cacao. No. 
25. INIAP-PROTECA. Quevedo. Ecuador. $143 \mathrm{p}$

Torres, J. (1995). Determinación de áreas de aptitud forestal para el establecimiento de plantaciones en el Litoral ecuatoriano. INEFAN, ITTO. Quito, EC. 185 $\mathrm{p}$

Waring, RH., and Running, SW. (1998). Forest Ecosystems "Analysis at Multiple Scales". Second Edition. San Diego, USA: Academic Press, pp. 19-57 